\title{
Letters
}

\section{Comment to: Gambelunghe G., Ghaderi, Cosentino A et al. (2000) Association of MHC Class I chain-related A (MIC-A) gene polymorphism with Type I diabetes. Diabetologia 43: 507-514}

To the Editor: We refer to the article by Gambelunghe et al [1] in which an association between Type I (insulin-dependent) diabetes mellitus and polymorphisms in the $M I C-A$ gene was found in a cohort of Italian patients. Several studies implicate a gene in the central MHC in susceptibility to Type I diabetes. The $M I C-A$ gene is a particularly interesting candidate given its high level of polymorphism and potential role in the regulation of $\gamma \delta$-T cells $[1,2]$. Gambelunghe and colleagues made two points. Firstly, $M I C-A 5$ was positively associated with Type I diabetes independently of $H L A-D R 3$ and/or $H L A$ $D R 4$ and with a very high odds ratio (approximately 172 when in association with $H L A-D R 3$ and/or $H L A-D R 4)$. Secondly, $M I C-A 5$ was associated with an earlier age of onset suggesting that the gene could affect disease progression. We find the re-

Table 1. $M I C$ - $A 5$ does not increase the risk of Type I diabetes in individuals carrying $H L A$ - $D R 3$ 'and/or' $H L A$ - $D R 4$

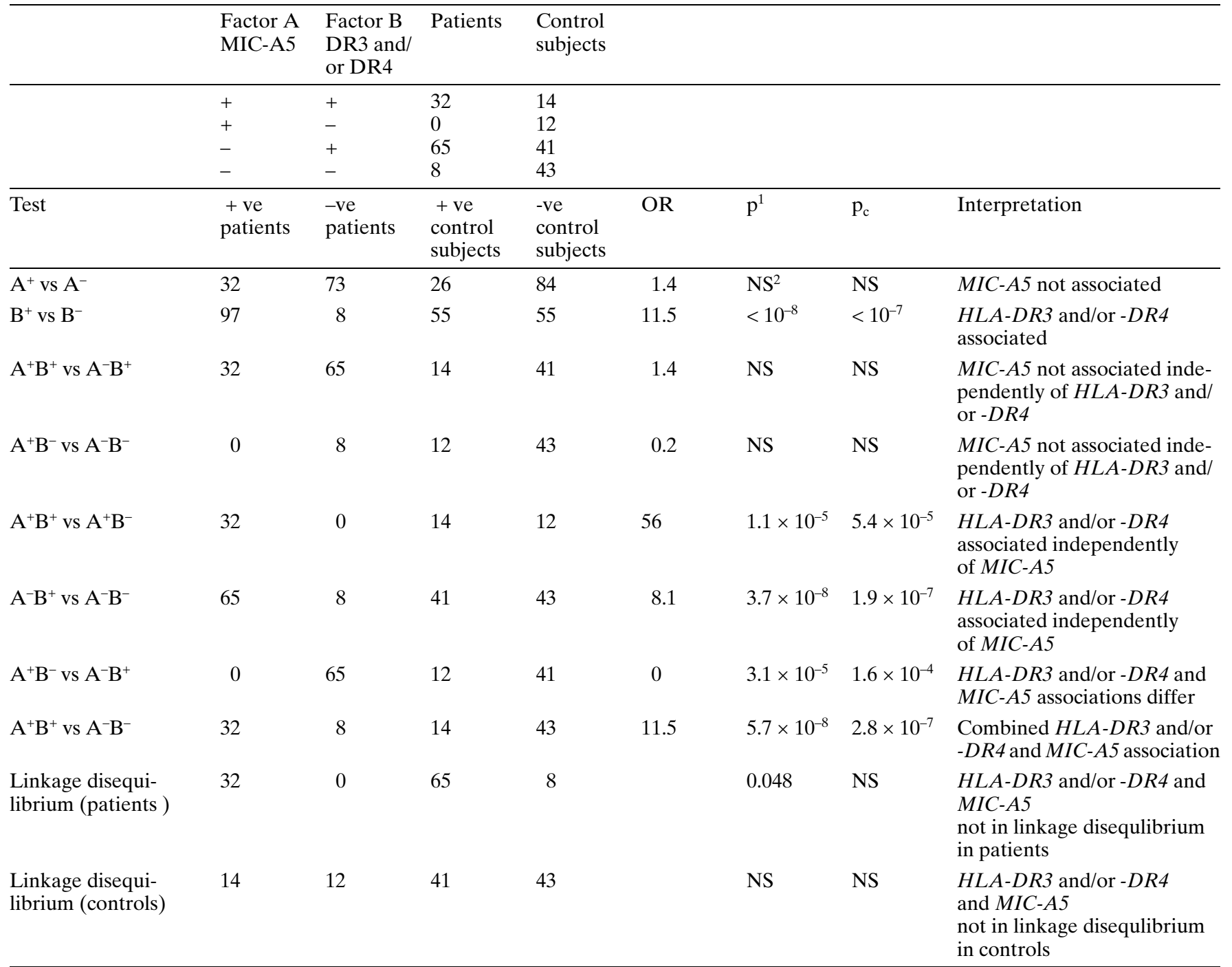

${ }^{1}$ Fischers exact test

${ }^{2} \mathrm{NS}=$ not significant

Corresponding author: R.J.N. Allcock, Department of Clinical Immunology and Biochemical Genetics, Royal Perth Hospital,Wellington Street, Perth 6001, Western Australia 
sults of Gambelunghe and colleagues very interesting and consistent with our studies and those of others which show that HLA class II alleles are the strongest indicator of risk for Type I diabetes, while central MHC genes modify this effect $[3,4,5]$.

We have, however, been unable to reproduce their findings in a cohort of 115 Type I diabetes patients and 110 control subjects in Western Australia. We found no association between any of the $M I C$ - $A$ transmembrane alleles and the disease (data not shown). When the cohort was stratified for the presence of either HLA-DR3 and/or HLA-DR4, there was no independent association with $M I C$ - $A 5$ (see Table 1 ). The odds ratio associated with carriage of $H L A-D R 3$ and/or $H L A-D R 4$ (11.5), was similar to that found by Gambelunghe et al (9.3). The odds ratio associated with the presence of both $M I C-A 5$ and either $H L A-D R 3$ and/or $H L A-D R 4$ did not, however, increase over that for $H L A-D R 3$ and/or $H L A-D R 4$.

These data suggest a difference in the central MHC region of diabetogenic HLA haplotypes in the Western Australian and Italian populations. It could be that $M I C-A 5$ is a marker of a susceptibility gene that is only found in the Italian population. It is not likely that the differences in the two studies are related to technical difficulties, as genotyping for $M I C-A$ transmembrane alleles is relatively simple. It will be important for $M I C$ - $A$ to be studied in other Italian and non-Italian countries to reconcile the difference between our studies and the studies of Gambelunghe et al. [1].

\section{R. Allcock, K. Cheong, F. Christiansen, C. Witt}

Acknowledgements. We would like to thank P. Price for reviewing the manuscript and V. McCann and T. Jones for providing access to patient samples. Manuscript number 2000-22 of the Department of Clinical Immunology and Biochemical Genetics, Royal Perth Hospital.

\section{References}

1. Gambelunghe G, Ghaderi, Cosentino A et al. (2000) Association of MHC Class I chain-related A (MIC-A) gene polymorphism with Type I diabetes. Diabetologia 43: 507-514

2. Fodil N, Laloux L, Wanner V et al. (1996) Allelic repertoire of the human MHC class I MICA gene. Immunogenetics 44: 351-357

3. Groh V, Rhinehart R, Secrist H, Bauer S, Grabstein KH and Spies T (1999) Broad tumour-associated expression and recognition by tumour-derived gamma delta T cells of MICA and MICB. Proc Nat Acad Sci USA 96: 6879-6884

4. Degli-Esposti MA, Abraham LJ, McCann V, Spies T, Christiansen FT, Dawkins RL (1992) Ancestral haplotypes reveal the role of the central $\mathrm{MHC}$ in the immunogenetics of IDDM. Immunogenetics 36: 345-356

5. Hanifi Moghaddam P, de Knijf P, Roep BO et al. (1998) Genetic structure of IDDM1: Two separate regions in the major histocompatibility complex contribute to susceptibility or protection. Diabetes 47: 263-269

\section{The authors' reply:}

To the Editor: R. Allcock et al. in their letter present interesting results on "MICA transmembrane polymorphism in Type I (insulin-dependent) diabetes mellitus in Western Australia“. Genotyping for the trinucleotide repeat polymorphism of MICA transmembrane region in 115 patients with Type I diabetes (T1DM) and 110 control subjects did not show any association with MICA alleles in their population. They also do not see any additional risk conferred by MICA over and above the risk conferred by high risk HLA-DR3 and/or DR4.

The reasons for their observation could be:

Firstly, that the population studied could be different. In this context if they would have given the frequencies of the MICA alleles in their healthy control subjects, it would be clear how different the population they studied looked. Unfortunately these results have not been shown.

Secondly, the control subjects were not matched with patients for age and sex. Their letter does not provide this information. We have shown below that different alleles of MICA are associated with T1DM in the young and T1DM occurring in the old. In addition, we have discussed different associations of MICA with T1DM in different ethnic groups, strengthening the importance of MICA as an additional genetic marker of the disease.

Thirdly, their T1DM patients are a mixed group of younger and older onset patients.

Accepted: 15 January 2001

Corresponding author: C.B. Sanjeevi, Department of Molecular Medicine, Karolinska Hospital, CMM, L8 03, S-17176 Stockholm, Sweden
We and others have studied MICA polymorphism and its association with T1DM in HBDI families from the United States, Koreans, Latvians, Asian Indians from India, Chinese and Japanese. In HBDI families, we found that MIC-A alleles 5 and 5.1 were transmitted from HBDI parents to children with T1DM significantly $(p<0.05)$ more frequently than expected, suggesting that MIC-A allele 5 and 5.1 are associated with susceptibility to T1DM in HBDI families [1]. In Latvians, an association of allele 5 was found to be associated with T1DM patients [2]. In Asian Indians, again allele 5 was associated with T1DM [3]. The association of alleles 5 in these populations was seen in T1DM patients who were young and younger than 18 years of age.

In the Korean patients with T1DM, allele 6 was significantly negatively associated [4]. In Japanese patients with T1DM, the frequency of allele A4 was significantly higher and that of alleles 6 was significantly lower $(p<0.05)$ [5]. The A4 allele was associated with susceptible haplotype DR4DQB1*0401 and A6 allele was associated with protective haplotype DR15-DQB1*0601 in Japanese. In the Spanish Basque families with T1DM, allele 4 was associated significantly positively with the disease similar to that seen in Japanese subjects and the presence of allele 9 was associated with protection $(p<0.05)[6]$. The Table summarizes the positive associations reported in different studies.

The association of allele 5 of MICA was observed only in T1DM patients of younger onset. In T1DM patients with onset over 25 years allele 5.1 was positively associated in Italian patients [7]. In the Japanese population, however, allele 5.1 was associated negatively in older onset patients [5]. The association of allele 5.1 was also observed in patients with Latent Autoimmune Diabetes in Adults (LADA) in Italian and Asian Indians [3].

We cannot rule out, however, the possibility that the association between MICA and autoimmune diabetes is due to a linkage disequilibrium with a still unidentified gene. If this is 